\title{
Erratum to: Therapy of HIV-associated lymphoma-recommendations of the oncology working group of the German Study Group of Physicians in Private Practice Treating HIV-Infected Patients (DAGNÄ), in cooperation with the German AIDS Society (DAIG)
}

\author{
Marcus Hentrich • Christian Hoffmann • \\ Franz Mosthaf • Markus Müller • Jan Siehl • \\ Christoph Wyen • Manfred Hensel
}

Published online: 1 May 2014

(C) Springer-Verlag Berlin Heidelberg 2014

Erratum to: Ann Hematol

DOI 10.1007/s00277-014-2058-4

The March 29, 2014, review article by Hentrich et al. entitled "Therapy of HIV-associated lymphoma - Recommendations of the oncology working group of the German Study Group of Physicians in Private Practice Treating HIV-Infected Patients (DAGNÄ), in cooperation with the German AIDS Society (DAIG)" (Ann Hematol, online March 29, 2014) contained an error. In the section "Diffuse large B-cell Lymphoma", line 3, at the end of the first sentence, the level of evidence was incorrectly stated. It should read "Six cycles of R-CHOP-21 are standard of care (AII)" instead of "Six cycles of R-CHOP21 are standard of care (AIII)". The authors apologize for the mistake.

The online version of the original article can be found at http://dx.doi.org/ 10.1007/s00277-014-2058-4.

M. Hentrich $(\bowtie)$

Department of Hematology, Oncology and Palliative Care,

Harlaching Hospital, Academic Teaching Hospital of the University

of Munich, Sanatoriumsplatz 2, 81545 Munich, Germany

e-mail: marcus.hentrich@klinikum-muenchen.de

C. Hoffmann

IPM Study Center, Hamburg and University of Schleswig Holstein,

Campus Kiel, Kiel, Germany

F. Mosthaf

Hematology, Oncology and Infectious Diseases Practice, Karlsruhe,

Germany

\section{Müller}

Department of Infectious Diseases, Vivantes

Auguste-Viktoria-Hospital, Berlin, Germany
J. Siehl

Hematology, Oncology and Infectious Diseases Practice, Ärzteforum Seestraße, Berlin, Germany

C. Wyen

First Department of Internal Medicine, University of Cologne, Cologne, Germany

M. Hensel

Hematology, Oncology and Infectious Diseases Practice, Mannheim, Germany 